\title{
Effect of Drawing on Structure and Properties of a Liquid Crystalline Polymer and Polycarbonate In-Situ Composite
}

\author{
QINGHUANG LIN, JAEYOUNG JHO, and ALBERT F. YEE \\ Department of Materials Science and Engineering \\ The University of Michigan \\ Ann Arbor, Michigan 48109
}

\begin{abstract}
Fibers (strands) with various draw ratios were spun from the liquid crystalline state of a pure aromatic liquid crystalline copoly(ester amide) and the melts of its blend with polycarbonate. Scanning electron microscopy (SEM), wide angle X-ray scattering (WAXS), and differential scanning calorimetry (DSC) were employed to investigate the structure and properties of the resulting fibers. Mechanical properties of the fibers were also evaluated. It was found that both the crystallite size and heat of fusion of the liquid crystalline polymer (LCP) increase steadily with draw ratio. However, the crystal-nematic transition temperature of the LCP is virtually unaffected by drawing. Moreover, heat of fusion of LCP is much smaller than that of isotropic condensation polymers despite the presence of very sharp diffraction peaks in WAXS measurements. These results are ascribed to the (semi)rigid rod nature of the LCP chains and the persistence of an ordered structure in the LCP melt, i.e., entropy effect. It was further observed that tensile modulus and tensile strength along fiber axis rise with draw ratio for the composite fibers. The elastic modulus of the composite fibers were found to be as high as $19 \mathrm{GPa}$ and tensile strength reached $146 \mathrm{MPa}$ with draw ratios below 40 and an LCP content of 30 wt\%. Compared with the thermoplastic matrix, the elastic modulus and tensile strength of the in-situ composite have increased by 7.3 times and 1.4 times, respectively, with the addition of only $30 \mathrm{wt} \% \mathrm{LCP}$. This improvement in mechanical properties is attributed to fibrillation of the LCP phase in the blend and the increasing orientation of the LCP chains along the fiber axis during drawing.
\end{abstract}

\section{INTRODUCTION}

$\mathrm{n}$ recent years, in-situ composites containing thermotropic liquid crystalline polymers (LCPs) have received increasing attention because of their scientific and technological importance (1-10). The premise behind the in-situ composite concept is the existence of an immiscible mixture of LCP and isotropic polymer (11), in which the dispersed LCP phase is deformed into a fibrillar form during processing, acting as a reinforcing phase (12). Consequently, significant improvements in physical properties could be achieved by using a small portion of LCP. In this sense, in-situ composites are akin to short fiber reinforced thermoplastic composites. However, LCP insitu composites have many advantages over conventional short fiber reinforced composites. The two most distinct advantages are: 1) The reinforcing fibrillar structure is formed in-situ, i.e., the formation of the reinforcing phase and the composite itself is completed in a single step. A separate process to produce the reinforcing fiber, as in short fiber reinforced com- posites, is unnecessary. Hence it is a potentially economical process to generate high performance materials; 2) LCP functions as a processing aid because of its extremely low melt viscosity, thereby making the use of high viscosity thermoplastics possible. Obviously, the in-situ composite concept offers a new method for tailoring physical properties of polymers without having to go through expensive chemical procedures. This has stimulated much research on insitu composites in academia as well as in industry.

In the past few years, much work has been done on in-situ composites, especially on the conditions for LCP fibrillation, since this is the key step for successful fabrication of in-situ composites. Although theoretical work has largely been limited to the small deformation of Newtonian or viscoelastic fluids (13-15), extensive experimental efforts have been devoted to the investigation of optimum conditions for LCP fibrillation. Many factors, such as material selection (1), composition (10,16-18), and a number of processing parameters, such as processing device (19), flow field $(17,20)$, viscosity and viscosity ratio 
$(17,21,22)$, processing temperature $(23,24)$, and interfacial tension (25-28), have been found to influence the morphology, and hence the properties, of the finished in-situ composite products. Among these factors, the flow field has been found to be of primary importance for a specific LCP/isotropic polymer system. Simple extensional flows, such as melt drawing, are most effective to produce LCP microfibrils and the effectiveness increases with draw ratio $(17,20)$. Recently, Sun, et al. (29), investigated the morphology, molecular orientation and mechanical properties of Ultem/Vectra in-situ composite films. Their qualitative WAXS results showed that the molecular orientation of LCP increases with drawing. They further found that the increased molecular orientation corresponds to an increase in mechanical properties. Unfortunately, a complete understanding of the effect of drawing on the structure and physical properties of in-situ composites is still lacking. The objective of this paper is, therefore, to investigate the structure of in-situ composites produced by variations in the draw ratio and correlate it to the physical properties of this novel material. To this end, a co-rotating twin-screw extruder was employed to fabricate in-situ composite strands using Vectra $\otimes B 950$ as the LCP phase and a bisphenol-A polycarbonate (PC) as the matrix. Pure LCP fibers were also spun from the liquid crystalline state. Scanning electron microscopy (SEM), differential scanning calorimetry (DSC), and wide angle X-ray scattering (WAXS) were applied to probe the structure and properties of the resulting strands. Mechanical properties of the strands were also evaluated. The effect of drawing on the structure and physical properties of in-situ composites is systematically investigated.

\section{EXPERIMENTAL}

\section{Materials}

The LCP used in this research was Vectra $\&$ B950 purchased from Hoechst-Celanese. According to the manufacture, Vectra $B 950$ LCP is a random copoly(ester amide) consisting of three components: 2,6-hydroxynaphthoic acid, terephthalic acid, and para-aminophenol. Its molecular weight is greater than 20,000 (30). The exact composition has not been officially disclosed by the manufacturer. Some researchers $(31,32)$ speculated that Vectra $\mathrm{B950}$ consists of $60 \%$ 2,6-hydroxynaphthoic acid, $20 \%$ terephthalic acid and $20 \%$ para-aminophenol. The matrix thermoplastic material utilized was a Lexan $\mathbb{B}$ bisphenol-A polycarbonate (PC) (Lexan 151), which was kindly supplied by the General Electric Co. The pellets of LCP and PC were dried in an air-circulating oven at $120^{\circ} \mathrm{C}$ for at least $24 \mathrm{~h}$ prior to processing. The polycarbonate/Vectra $\mathrm{B950}$ blends contained 30 wt\% LCP (hereafter referred to as PC/V30).

\section{Processing}

Composite strands (fibers) were produced by mixing pellets of the LCP and PC in the dry state, feed- ing the mixture through an extruder, and drawing the resulting strand. The extruder was a Werner \& Pfleiderer 30-mm co-rotating twin-screw extruder (ZSK-30) with a $3.2 \mathrm{~mm}$ conical die. The downstream barrel temperatures were set at $280^{\circ} \mathrm{C}$. The extruded molten strand was either collected in an undrawn form, or led through a set of take-up reels to draw the strand. Typical drawn strand diameters ranged from 0.5 to $2 \mathrm{~mm}$. The pure Vectra fibers were spun from the liquid crystalline state with an Instron capillary rheometer at $290^{\circ} \mathrm{C}$. Draw ratio is defined as the ratio of the cross-section areas of the die to the extruded strand.

\section{SEM}

Morphologies of the extruded strands were obtained by examining the fracture surfaces with a Hitachi S-800 scanning electron microscope with low working voltages (less than $5 \mathrm{KV}$ ). Drawn strands were fractured in tension and undrawn strands in flexure, both at room temperature. To observe individual microfibrils, the PC matrix was extracted with chloroform. All SEM samples were sputter coated with a thin layer of carbon to avoid charging.

\section{DSC}

Differential scanning calorimetry measurements were conducted on a Perkin-Elmer thermal analysis system (DSC-7) under a nitrogen atmosphere. Sample weights ranged from 10 to $20 \mathrm{mg}$. Heating rate was $20^{\circ} \mathrm{C} / \min$. Peak temperatures were taken as crystal-nematic transition temperatures or melting temperatures. In cases of blend samples, the heat of fusion of LCP was obtained by normalizing the measured value over the weight fraction of LCP in the blend.

\section{WAXS}

Wide angle $X$-ray diffraction patterns were taken on a Rigaku Rotaflex 200-BX X-ray diffractometer with a focusing monochromator in the diffracted beam and a scintillation registration. The tube voltage was 40 $\mathrm{KV}$ and tube current $100 \mathrm{~mA}$. Meridional scans and equatorial scans were taken in a continuous transmission mode with a scanning speed of $1 \%$ min and a sampling interval of $0.01^{\circ}$. The orientation parameter was calculated from measurements using a pole figure attachment. Azimuthal scans were run on the pole figure attachment in a stepwise symmetric transmission mode with a step of $1^{\circ}$ and a count time of 5 or $8 \mathrm{~s}$. Unless otherwise specified, all azimuthal scans were taken at $2 \theta=43^{\circ}$, which is the strongest peak in the meridional scans. Background scattering was taken at $2 \theta=50^{\circ}$ and was subtracted from the overall intensity for orientation parameter calculations.

\section{Mechanical Property Measurement}

Mechanical properties of the extruded strands were measured on an Instron Mechanical Tester (Model 
4502). Elongation was determined with the aid of 0.5 -inch $50 \%$ extensometer attached directly to the specimen. The gage length was kept constant at 100 mm for each measurement. To reduce stess concentration at the grips, specimens were held by using rubber pads on the serrated grips. All tests were run at a constant crosshead speed of $0.5 \mathrm{~mm} / \mathrm{min}$. All data were acquired by an IBM computer and mechanical quantities were calculated by a built-in computer program. All data points were the average of at least three tests. Standard deviations were less than $10 \%$.

\section{RESULTS AND DISCUSSION}

\section{Effect of Drawing on Morphology}

The development of a fibrillar structure is a key step to achieving reinforcement. However, the generation of an LCP fibrillar structure is a complex rheological as well as thermal process $(24,33)$. In order to obtain the fibrillar structure, the dispersed LCP phase must be deformed in an appropriate flow field and frozen into the solid state before it could relax back to the spherical form. We have conducted extensive research on the conditions for LCP fibril formation (34). It has been found that the morphology of the finished composite strands depends on the material selection, composition of the components, and a number of processing parameters, such as mixing device, screw configuration, extruder barrel temperature, screw speed, material feed rate, and drawing. Our experimental results showed that drawing as well as screw configuration design are crucial to achieving a welldefined LCP microfibril morphology. The viscosity and viscosity ratio are two other important parameters in controlling the deformation of the LCP droplets. Through careful control of the processing conditions, composite strands with a uniform distribution of LCP phase and a well-defined LCP fibrillar morphology have been successfully fabricated. A more detailed discussion of the processing parameters and their effects on resultant morphology is the subject of a forthcoming paper (34).

The effect of drawing on morphology is shown in the SEM micrographs of the fracture surfaces of a drawn and an undrawn strand of PC/V30 blend (Fig. 1). It is seen that the dispersed LCP phase appears essentially in a spherical form on the fracture surface of the undrawn sample, while the sample with a draw ratio of 25 shows uniform and well-defined LCP microfibrils. Upon extraction of the PC matrix with chloroform, LCP microfibrils are found to be free of irregular surfaces and small undeformed particles. The diameter of the microfibrils is in the range of 0.05 to $0.3 \mu \mathrm{m}$ (Fig. 2).

Theoretical treatment of the deformation of a dispersed LCP phase in an isotropic medium has been lacking. Taylor's theory on the deformation of Newtonian droplets suspended in another Newtonian fluid has frequently been used to explain the morphology of in-situ composites even though this theory is strictly applicable only to small deformations. Taylor

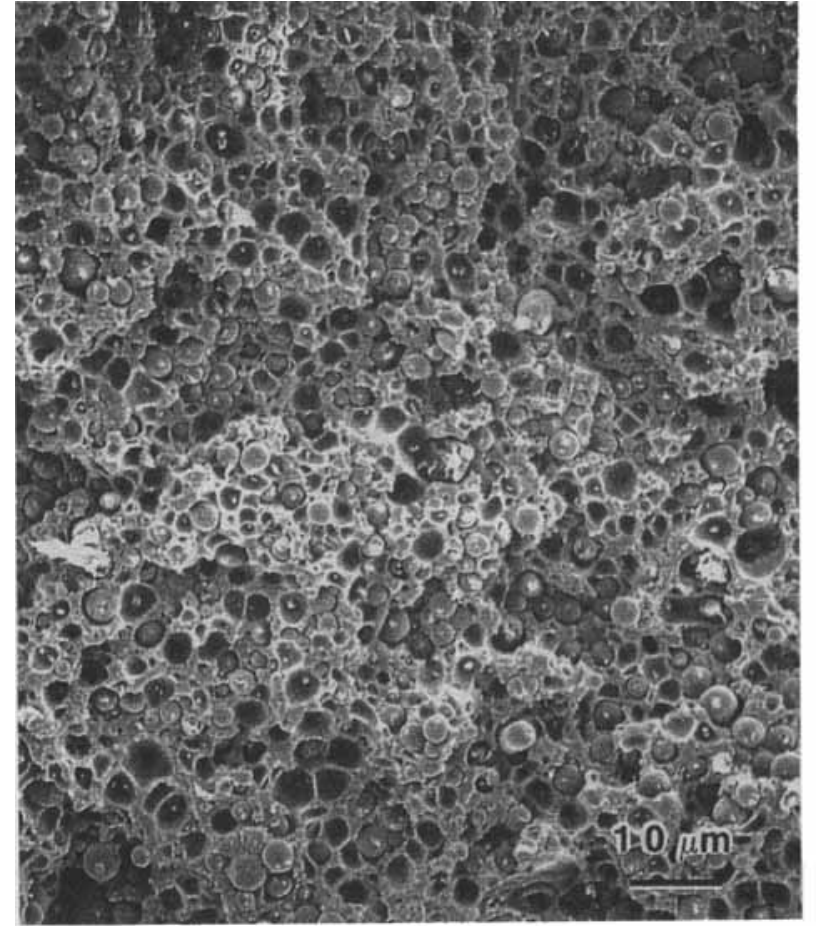

(a)

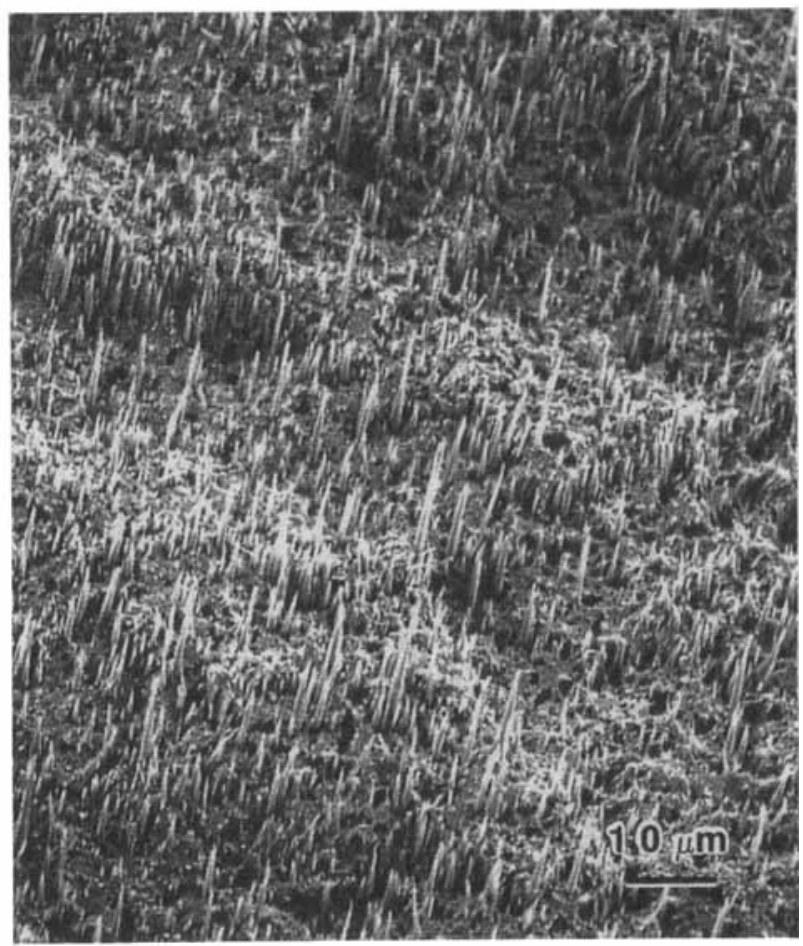

(b)

Fig. 1. SEM micrographs of the fracture surfaces of an undrawn (a) and a drawn $(D R=25)$ (b) strand of $P C / V 3 O$ blend.

(13) showed that an initially spherical droplet will be deformed into an ellipsoid under uniform shearing of plane hyperbolic flow field, depending on the viscosity ratio and Webber number (We). When interfacial ten- 


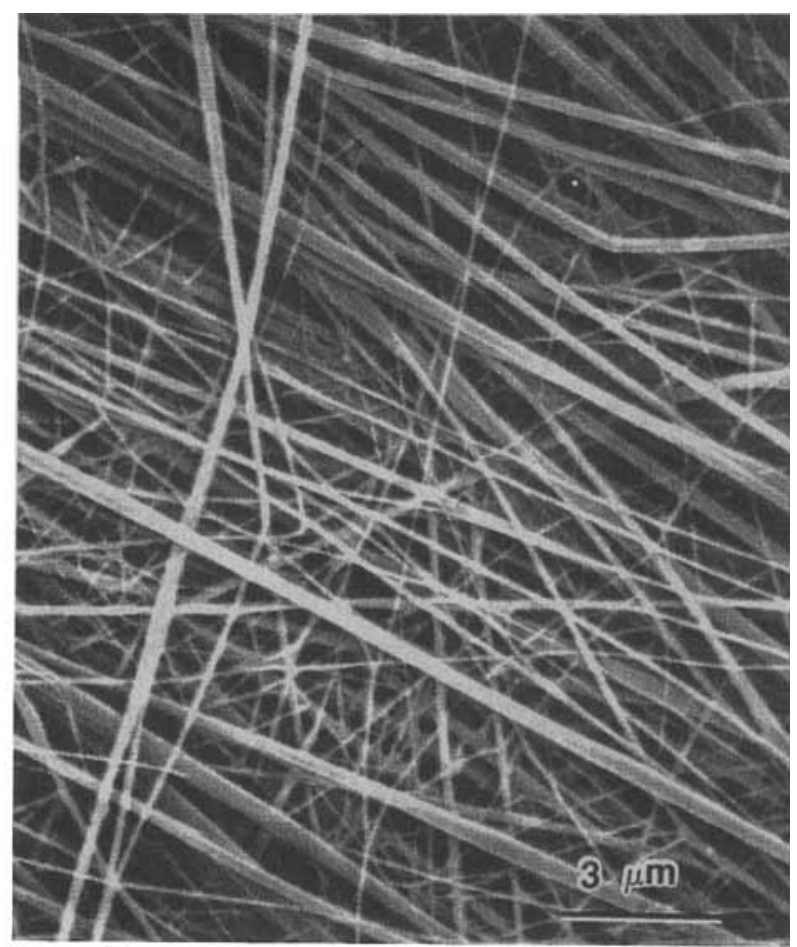

Fig. 2. SEM micrograph of the microfibrils in a drawn sample after extracting the PC matrix.

sion effect dominates viscous effect, the apparent deformation $D$ is given as:

$$
D=W e \frac{19 K+16}{16 K+16}
$$

Where

$$
K=\frac{\eta_{D}}{\eta_{m}}, \quad D=\frac{L-B}{L+B}, \quad W e=\frac{\eta_{m} \dot{\gamma} a}{\sigma}
$$

L, B are the major and minor axis of the ellipsoid, respectively;

$\eta_{D}, \eta_{m}$ are viscosities of dispersed phase and suspending phase, respectively;

$\dot{\gamma}$ is shear rate;

$a$ is the radius of the dispersed phase; and

$\sigma$ is interfacial tension.

Here We number can be considered as the ratio of the deforming force $\left(\eta_{m} \dot{\gamma} a\right)$ to the surface tension force $(\sigma)$ that tends to keep the dispersed phase in the spherical form. Drawing increases the deforming force, thus leading to a higher aspect ratio. In other words, drawing is conducive to LCP microfibril formation. In fact, our experimental results show that given sufficiently large LCP droplets, the LCP phase can be readily stretched into the fibrillar form by drawing. These findings are in good agreement with those obtained by other research groups $(17,20)$.

\section{Effect of Drawing on Crystalline Structure of LCP}

The effect of flow or external stress (strain) on polymer crystallization has been known to exist in many isotropic polymer systems $(35,36)$. Flow or external stress promotes molecular orientation. The chains oriented into an extended chain conformation in a melt, a glassy state, or a solution facilitate their own nucleation and crystallization. As a result, the crystallization rate is significantly enhanced, and the melting temperature of the resultant crystal is increased. This phenomenon is referred to as flow-induced crystallization or stress-induced crystallization. When it occurs, the orientation of polymer chains is preserved in the crystalline state. We report first this stress-induced crystallization in an anisotropic polymer system and its blend with an isotropic polymer.

Figures 3 and 4 show representative meridional and equatorial WAXS patterns of pure Vectra fibers. Comparing diffraction patterns of undrawn and drawn samples, it is obvious that drawing significantly improves the crystal structure of LCP. The undrawn sample exhibits a relatively sharp peak at $2 \theta=43^{\circ}$, and five diffuse peaks at $2 \theta=9.4^{\circ}, 11.4^{\circ}$, $19.2^{\circ}, 21.0^{\circ}$, and $30.6^{\circ}$ on meridional scans. The peak at $2 \theta=43^{\circ}(d=2.1 \AA)$ represents the diffraction from the crystalline planes perpendicular to the chain axes, i.e., the c-axis (37). At a draw ratio of 2.5 , the diffraction peak at $2 \theta=19.2^{\circ}$ disappears; the diffraction

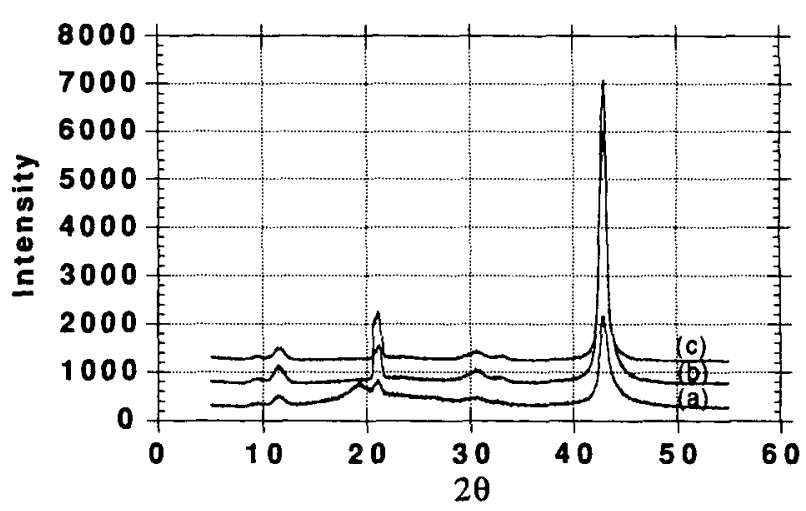

Fig. 3. Meridional WAXS patterns of Vectra fibers with vari ous draw ratios (a) $D R=1.0$, (b) $D R=2.5$, (c) $D R=10.0$.

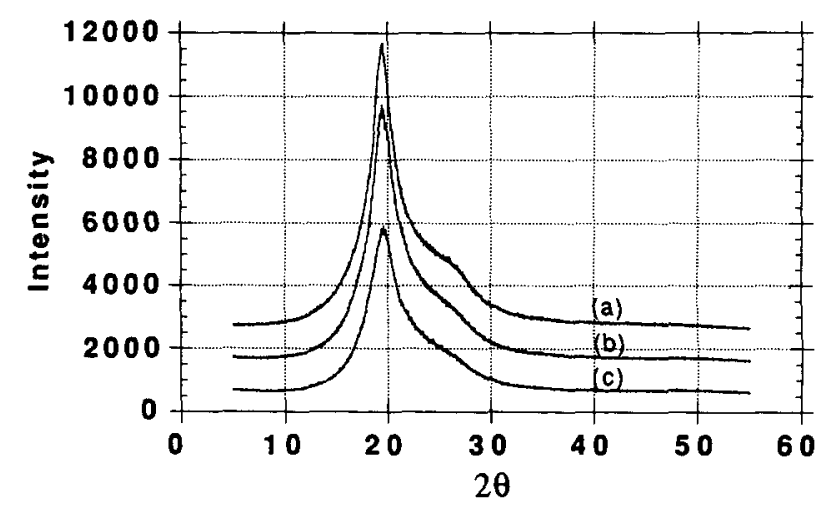

Fig. 4. Equatorial WAXS patterns of Vectra fibers with vari ous draw ratios (a) $D R=1.0$, (b) $D R=10.0$, (c) $D R=31.9$. 
peak at $21.0^{\circ}$ becomes sharper, and a new diffraction peak is detected at $2 \theta=33.0^{\circ}$. Furthermore, the relative intensity of the peak at $2 \theta=43^{\circ}$ is greatly enhanced. It is also noticed that the diffraction patterns do not change appreciably for samples with draw ratios greater than 2.5 . The equatorial scans show a single diffraction peak at $2 \theta=19.6^{\circ}(d=4.5 \AA)$. This peak corresponds to the interchain correlation of the LCP.

The crystallite size is calculated according to Scherrer's equation $(38,39)$ :

$$
L=\frac{0.9 \lambda}{\Delta(2 \theta) \cos \left(\theta_{0}\right)}
$$

Where $\lambda$ is the wave length of X-ray $\lambda=1.542 \AA$;

$\Delta(2 \theta)$ is the angular width at half maximum; and $\theta_{0}$ is the diffraction angle of the peak in question.

Here $2 \theta_{0}$ is taken to be $43^{\circ}$. Therefore, the crystallite size is the dimension along the polymer chain axis. It must be pointed out that crystal distortion and equipment factors may also contribute to the broadening of diffraction peaks. Therefore, the actual crystallite size is probably larger than the value given by the above equation. The change in crystallite size with draw ratio is plotted in Fig. 5. As expected, the apparent crystallite size of the LCP increases monotonously with draw ratio. A similar trend is observed in the blends of $30 \mathrm{wt} \%$ Vectra with polycarbonate (Fig. 6). These results suggest that drawing orients the LCP chains along the fiber direction, hence increasing the crystallite dimension of the LCP along chain direction.

\section{Effect of Drawing on Thermal Properties}

The effect of drawing on the crystallization of LCP both in the pure form and in the blend was revealed by DSC measurements. Typical DSC traces are given in Fig. 7. The changes in thermal properties with the draw ratio are depicted in Figs. 8 through 12. As can be seen, drawing has a similar effect on the thermal

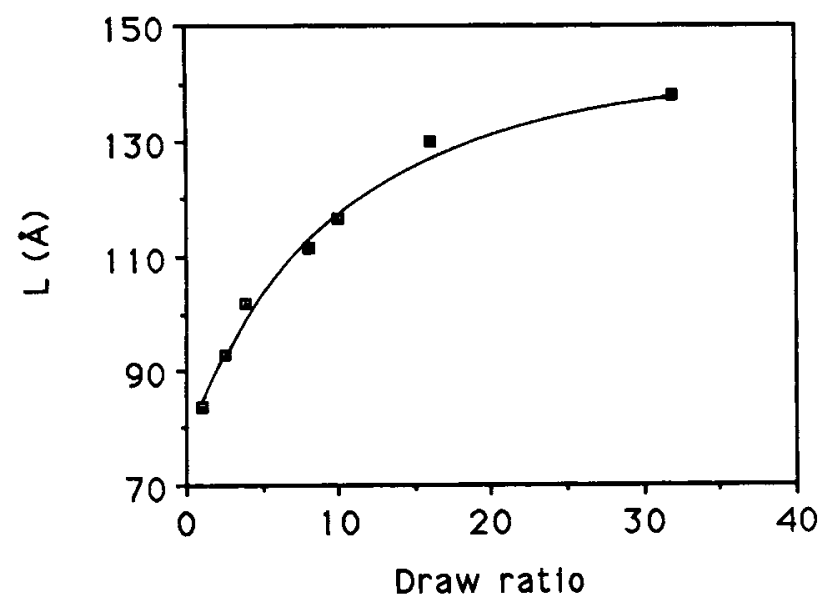

Fig. 5. Apparent crystallite size versus draw ratio for pure Vectra $B 950$ fibers.

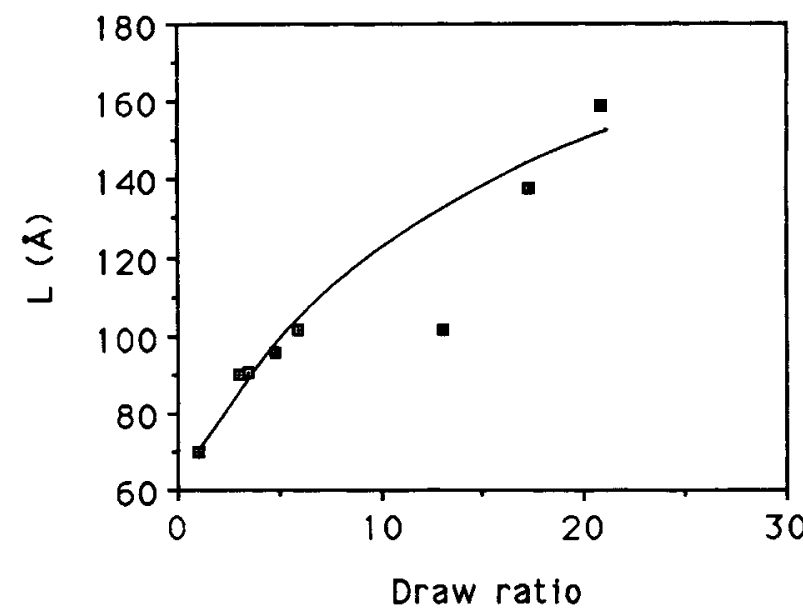

Fig. 6. Apparent crystallite size of $L C P$ versus draw ratio for Lexan 151 / Vectra B950 (30 wt\% LCP) composite strands.

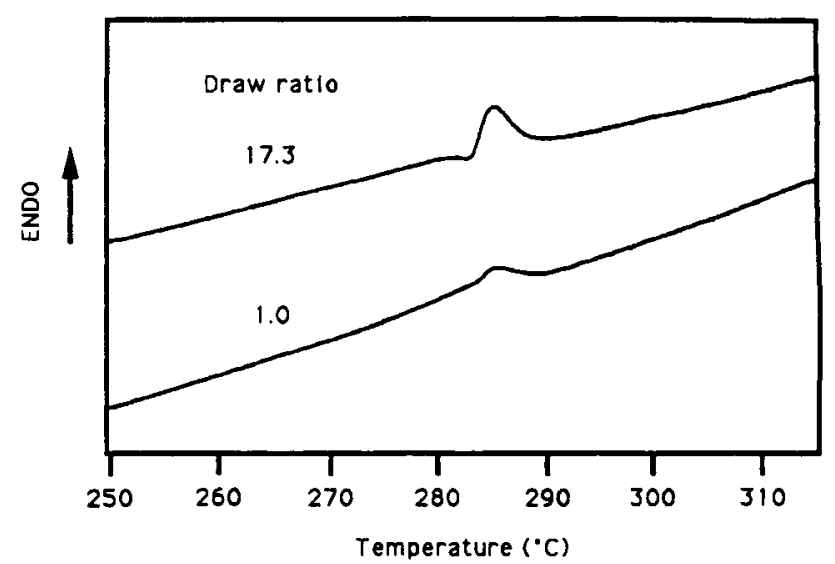

Fig. 7. Normalized DSC curves of the Lexan 151/Vectra $B 950$ (30 wt\% LCP) composite strands with different draw ratios.

properties of the LCP in both pure form and in the blends. The heat of fusion of LCP increases continually with the draw ratio. However, the crystal-nematic transition temperature $T_{m}$ of LCP and the glass transition of the PC matrix are virtually unaffected by drawing. The invariance of glass transition temperature with drawing indicates that the drawing process used in the present study does not bring about any change in the structure of the glassy PC matrix.

It has been proposed (40) that the phase transition from the liquid to crystalline state of random-coiled chains is very much modified if the liquid phase is strained. The equilibrium melting or crystallization temperature $T_{m}$ for a strained system is expressed as:

$$
T_{m}=\frac{\Delta H-\delta H}{\Delta S-\delta S}
$$

Here $\Delta H$ and $\Delta S$ are, respectively, the enthalpy and entropy changes upon melting or crystallization when the system is strain free. The enthalpy decrease $(\delta H)$ results from better packing of the strained liquid phase, which reduces the distance between adjacent 


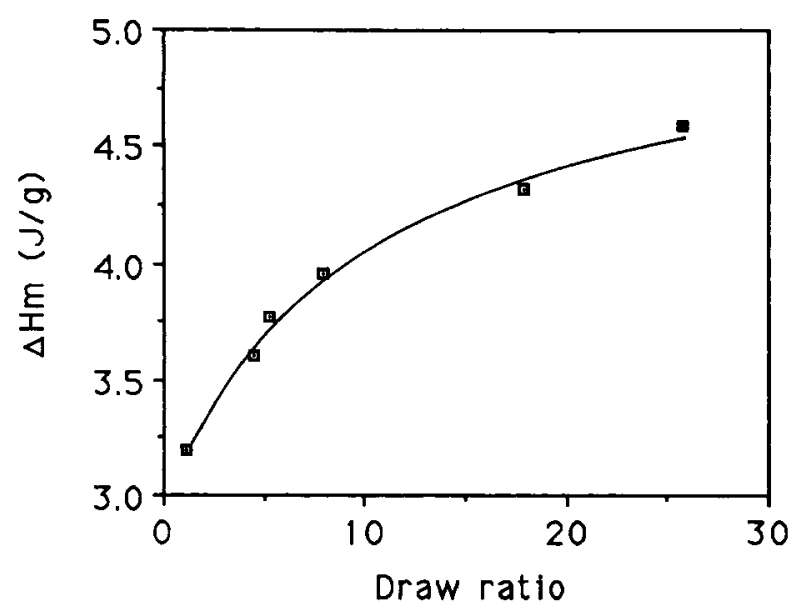

Fig. 8. Variation of heat of fusion with draw ratio for pure Vectra $B 950$ fibers.

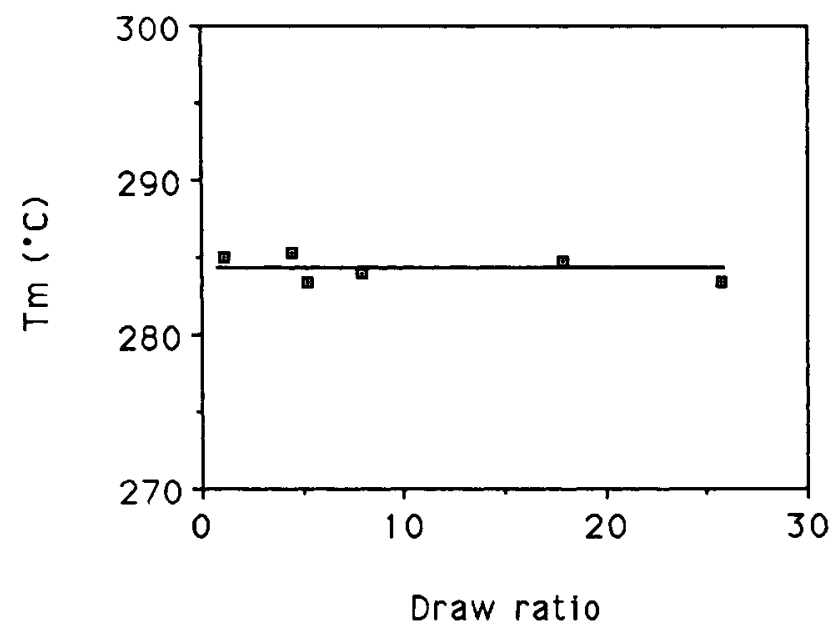

Fig. 9. Relationship between melting temperature and draw ratio for pure Vectra $B 950$ fibers.

chains, and increases the fraction of the lower energy trans conformation. The entropy decrease $(\delta S)$ is the consequence of a reduction of available conformations with the chain extension and orientation in the strained liquid phase. It was further shown that the equilibrium melting or crystallization temperature $T_{m}$ of the strained melt is always higher than that of the unstrained one. In other words, chain extension and orientation are responsible for the increase in melting temperature.

However, our experimental results show that, within experimental error, the melting temperature of LCP remains practically constant with the draw ratio (Figs. 9 and 11). This inconsistency with the above equation is not surprising if one considers the rigidity of the polymer under study and the oriented structure of the liquid crystalline melt. Vectra is known as a wholly aromatic copoly(ester amide), i.e., its polymer backbone consists solely of aromatic rings, specifically benzene rings and naphthalene rings,

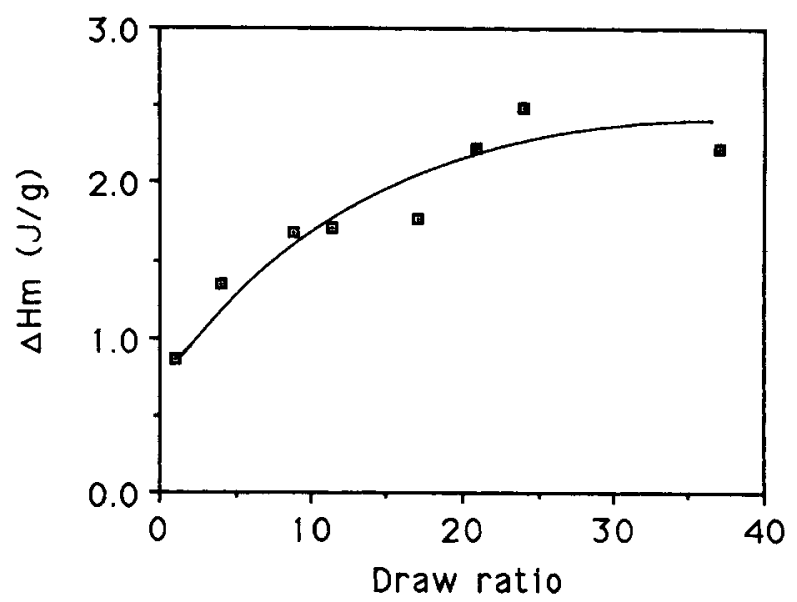

Fig. 10. Variation of heat of fusion of $L C P$ with draw ratio for Lexan 151 / Vectra B950 (30 wt\% LCP) composite strands.

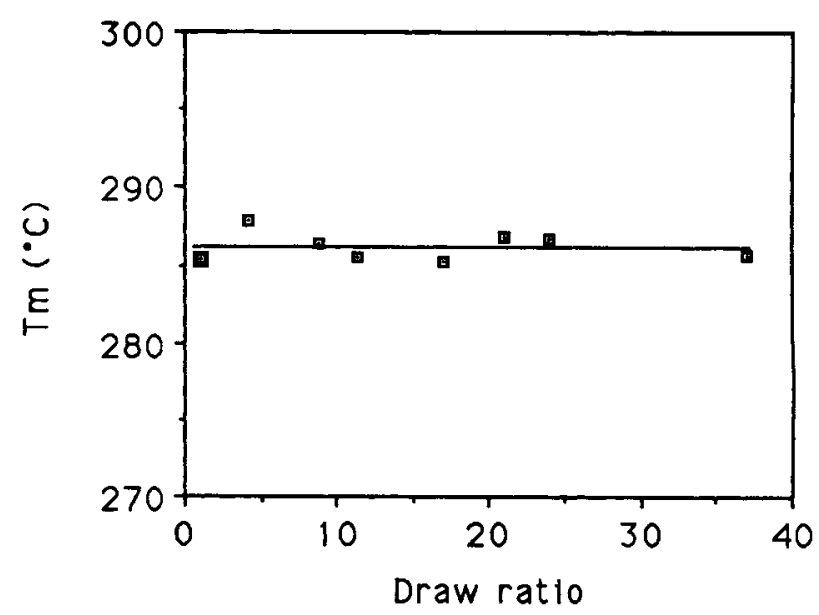

Fig. 11. Relationship between melting temperature of $L C P$ and draw ratio for Lexan 151/Vectra B950 (30 wt\% LCP) composite strands.

joined together by ester and amide linkages. Owing to this wholly aromatic nature of Vectra, the change in extension of this (semi)rigid chain during drawing is much smaller than that possible with the flexible chains, to which the proposed theory is applied. In addition, for liquid crystalline melts, local order exists even if they are unstrained. Consequently the entropy decrease $(\delta S)$ due to drawing for LCP is not as significant as for flexible polymers, thus keeping the crystal-nematic transition temperature independent of the draw ratio.

The increase in heat of fusion can be explained in terms of the entrophy change with draw ration. For an equilibrium phase transition, the heat of fusion $\Delta H_{m}=T_{m} \Delta S_{m}$. As will be shown later in this paper, the solid state of the LCP phase becomes more ordered as the draw ratio increases. As a result, the entropy change on melting increases with draw ratio, thus leading to an increase in the heat of fusion. It is also observed that, in spite of the presence of 


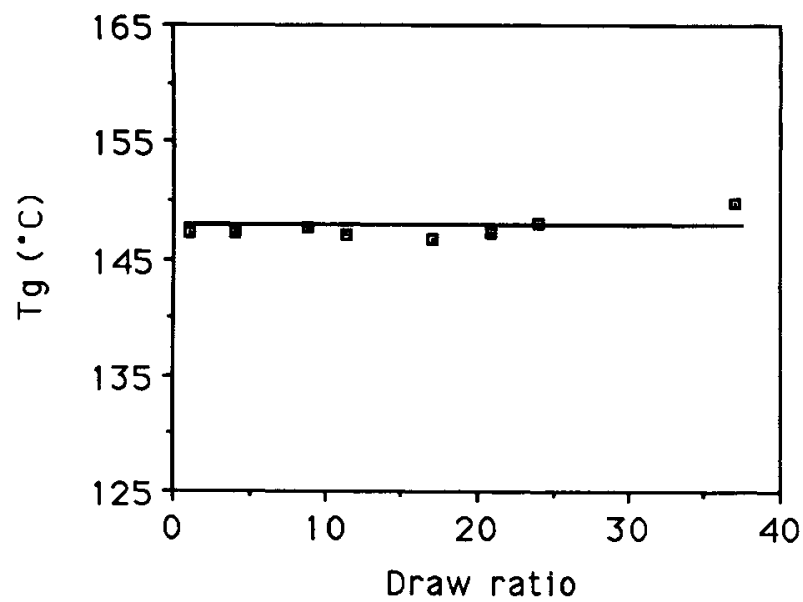

Fig. 12. Change of glass transition temperature of $P C$ with draw ratio for Lexan 151 / Vectra B950 (30 wt\% LCP) composite strands.

relatively sharp diffraction peaks in the meridional and equatorial scans, DSC measurements reveal an extremely low heat of fusion for the LCP crystal as compared with other condensation polymers. The heat of fusion is less that $5.0 \mathrm{~J} / \mathrm{g}$, which is far less than that of other condensation polymers [for example, the heat of fusion for PET is about $130 \mathrm{~J} / \mathrm{g}(41)]$. The reason again is ascribed to the ordered liquid crystalline melt. The ordered liquid crystalline melt gives rise to a lower value of $\Delta S_{m}$. It then follows that the heat of fusion for LCP is much lower that of isotropic polymers. The heat of fusion of LCP in pure form is always greater than that in the blends, suggesting that the stress-induced crystallization effect for the LCP is not as prominent in the blends as in its pure form. The reason is that the external stress is not applied directly to the LCP in the blends as is in pure LCP. Part of the external stress is relaxed during its transferring from the PC matrix to the LCP phase. It is also possible that the presence of the amorphous PC impairs the nucleation and the growth processes during LCP crystallization.

\section{Effect of Drawing on Molecular Orientation of LCP}

One of the assumptions of the in-situ composite concept is that the mechanical properties of oriented LCP are inherently superior to those of isotropic polymers. However, superior mechanical properties of LCP could not be achieved without high molecular orientation. Indeed, Chung (42) and Kenig (43) have shown that the tensile modulus of pure LCP increases with the Herman's orientation parameter. Therefore, the orientation parameter is one of the most important parameters in determining mechanical properties of in-situ composites.

The orientation parameter, $P_{2}$, is defined by the following equations $(44,45)$ :

$$
P_{2}=\frac{1}{2}\left(3\left\langle\cos ^{2} \alpha\right\rangle-1\right)
$$

$$
\left\langle\cos ^{2} \alpha\right\rangle=\frac{\int_{0}^{\pi / 2} I(\alpha) \sin \alpha \cos ^{2} \alpha d \alpha}{\int_{0}^{\pi / 2} I(\alpha) \sin \alpha d \alpha}
$$

where $\alpha$ is the angle between fiber axis and the director of orienting unit, and $I(\alpha)$ is the azimuthal intensity distribution. For a perfectly oriented sample, the value of orientation parameter, $P_{2}$, is 1 , whereas $P_{2}$ would equal zero for a sample with a random orientation.

Crevecoeur and Groeninckx (31) applied WAXS to determine the orientation parameter of LCP in blends of Vectra with polystyrene and a PPO/PS blend. It was found that Herman's orientation parameter was constant $(0.88)$ for strands with draw ratios ranging from 6 to 40 . This result was found for both pure Vectra B950 and a blend of Vectra A950/polystyrene containing $25 \%$ Vectra. Nonetheless, the orientation parameter increased with the LCP content. Similar results were reported for blends of 60PHB/40PET and polycarbonate (46). However, Chung (47) found that the orientation parameter was independent of composition but was dependent on draw ratio for Vectra/nylon 12 blends. More recently, Ajji, et al. (48), employed the wide angle X-ray scattering technique to measure the molecular orientation of LCP and the isotropic polymer in in-situ composites based on PBT and 60PHB/40PET. It was found that the orientation parameter of the isotropic poly(butylene terephthalate) (PBT) matrix increased with drawing. Without much experimental evidence, it was speculated that the orientation of the LCP phase is responsible for the increase in mechanical properties of the oriented blends.

The samples used in the present study were PC /V30 composite strands with draw ratios of $1,3.5$, $4.8,7.1,13.0,17.3$, and 20.8. Results of meridional and equatorial scans are summarized in Table 1 . With the exception of the undrawn sample $(D R=1.0)$, consistent diffraction patterns are obtained for all the samples. Furthermore, the relative intensity of the peak at $2 \theta=43^{\circ}$ on meridional scans increase with draw ratio. The scattering peak at $2 \theta=43^{\circ}$ on meridional scans is related to the intramolecular correlation of the LCP (37). Therefore azimuthal scans were taken at $2 \theta=43^{\circ}$ in order to measure the orientation of polymer chains along the fiber axis. In other words, the director of the orienting unit is along the LCP chain direction. Figure 13 gives experimental results of the variation of orientation parameter with draw ratio. It is seen that the orientation parameter rises sharply with the draw ratio, which then levels off at the draw ratio of about 10 . This trend corresponds well with the theoretical prediction based on a simple geometric model assuming a rigid-rod conformation for the LCP chains (49). It is the molecular orientation of the LCP chains that gives rise to the reinforcement effect in in-situ composites. The asymptotic value of the molecular orientation parameter is 0.75 . This result agrees very well with the one found for the blends of a polyetherimide and a liquid crystalline polymer (GE Ultem/K161) (50). 
Table 1. WAXS Results of PC/V30 Composite Strands.

\begin{tabular}{|c|c|c|c|c|c|c|c|c|}
\hline & & \multicolumn{7}{|c|}{ Draw Ratio } \\
\hline & & 1.0 & 3.5 & 4.8 & 7.1 & 13.0 & 17.3 & 20.8 \\
\hline \multirow{5}{*}{$\begin{array}{l}\text { Meridional } \\
\text { scan }\end{array}$} & $2 \theta\left(^{\circ}\right)$ & & 11.69 & 11.73 & 11.74 & 11.58 & 11.47 & 11.56 \\
\hline & $\begin{array}{c}d_{\text {hkl }}(\AA) \\
2 \theta\left(^{\circ}\right)\end{array}$ & 17.09 & $\begin{array}{r}7.56 \\
17.09\end{array}$ & $\begin{array}{r}7.54 \\
17.09\end{array}$ & $\begin{array}{r}7.53 \\
17.19\end{array}$ & $\begin{array}{r}7.64 \\
16.67\end{array}$ & $\begin{array}{r}7.71 \\
16.82\end{array}$ & $\begin{array}{r}7.65 \\
16.76\end{array}$ \\
\hline & $\begin{array}{c}d_{h k 1}(\AA) \\
2 \theta\left(^{\circ}\right)\end{array}$ & $\begin{array}{r}5.18 \\
18.99\end{array}$ & $\begin{array}{r}5.18 \\
20.93\end{array}$ & $\begin{array}{r}5.18 \\
20.87\end{array}$ & $\begin{array}{r}5.15 \\
20.93\end{array}$ & $\begin{array}{r}5.28 \\
20.95\end{array}$ & $\begin{array}{r}5.27 \\
21.05\end{array}$ & $\begin{array}{r}5.29 \\
21.04\end{array}$ \\
\hline & $\begin{array}{c}d_{h k 1}(\AA) \\
2 \theta\left(^{\circ}\right)\end{array}$ & $\begin{array}{r}4.67 \\
43.00\end{array}$ & $\begin{array}{r}4.24 \\
43.02\end{array}$ & $\begin{array}{r}4.25 \\
43.01\end{array}$ & $\begin{array}{r}4.24 \\
43.07\end{array}$ & $\begin{array}{r}4.24 \\
43.00\end{array}$ & $\begin{array}{r}4.22 \\
42.99\end{array}$ & $\begin{array}{r}4.22 \\
42.89\end{array}$ \\
\hline & $\mathrm{d}_{\mathrm{hkl}}(\AA)$ & 2.10 & 2.10 & 2.10 & 2.10 & 2.10 & 2.11 & 2.11 \\
\hline Equatorial & $2 \theta\left(^{\circ}\right)$ & 19.31 & 19.33 & 19.27 & 19.26 & 19.43 & 19.32 & 19.42 \\
\hline scan & $d_{h k \mid}(\AA)$ & 4.59 & 4.59 & 4.60 & 4.60 & 4.56 & 4.59 & 4.57 \\
\hline
\end{tabular}

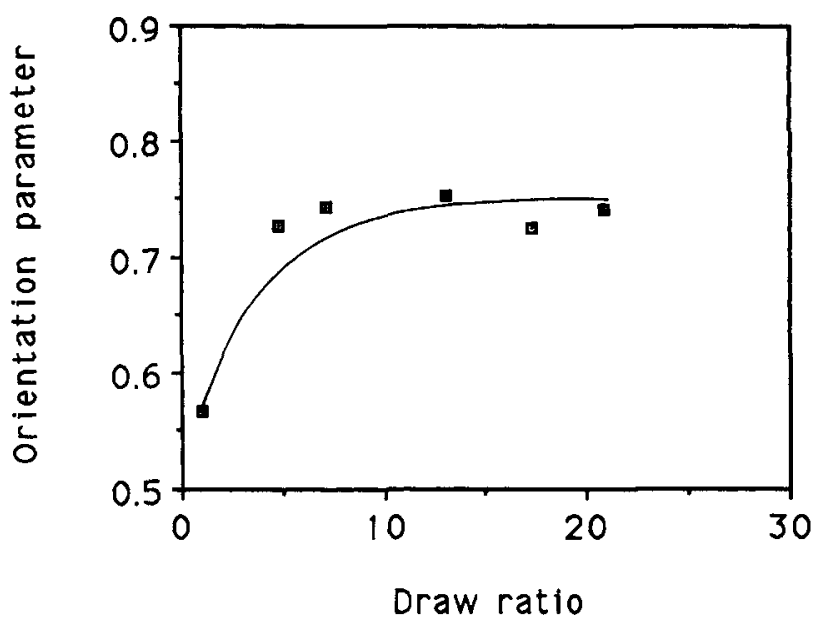

Fig. 13. Orientation parameter as a function of draw ratio for Lexan 151/ Vectra B950 (30 wt\% LCP) composite strands.

\section{Effect of Drawing on Mechanical Properties}

Variations of elastic modulus and tensile strength with draw ratio for PC/V30 strands are shown in Figs. 14 and 15. As can be seen, the elastic modulus increases steadily with increasing draw ratio up to 35. The elastic modulus of the strand with a draw ratio of 35 is more than three times that of the undrawn one. The highest value of elastic modulus is $19 \mathrm{GPa}$ for the composite strands. This is not surprising in view of the dependence of the elastic modulus of the LCP on drawing $(42,43)$. These results demonstrate that the elastic modulus can be markedly improved by drawing. Inasmuch as the elastic modulus of LCP fiber is generally several times greater than that of the PC matrix, the elastic modulus of in-situ composites is essentially determined by the modulus of the LCP reinforcing phase, according to the Halpin-Tsai equation (12). It then follows that the key to improving the modulus of in-situ composites is to promote LCP molecular orientation, although there are other factors affecting the modulus of in-situ composites such as the aspect ratio of the LCP microfibers.

As is shown in Fig. 15, the tensile strength of the strands with $30 \mathrm{wt} \%$ LCP rises sharply with increas-

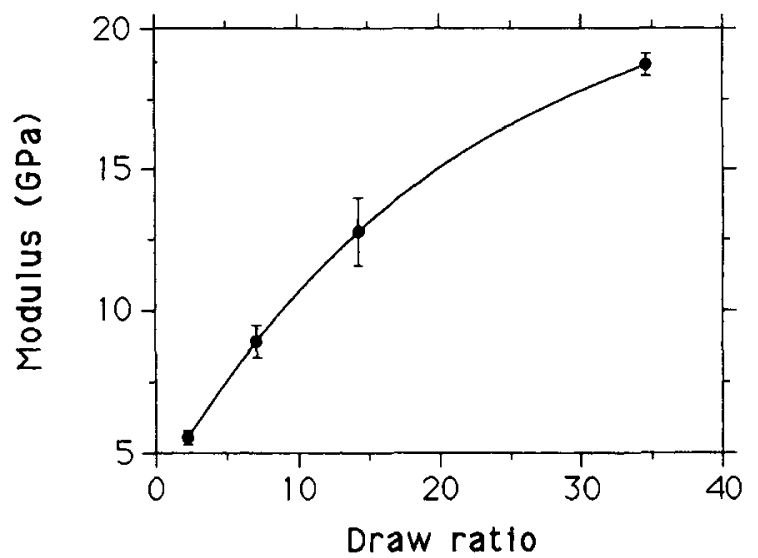

Fig. 14. Variation of elastic modulus with draw ratio for Lexan 151 / Vectra B950 (30 wt\% LCP) composite strands.

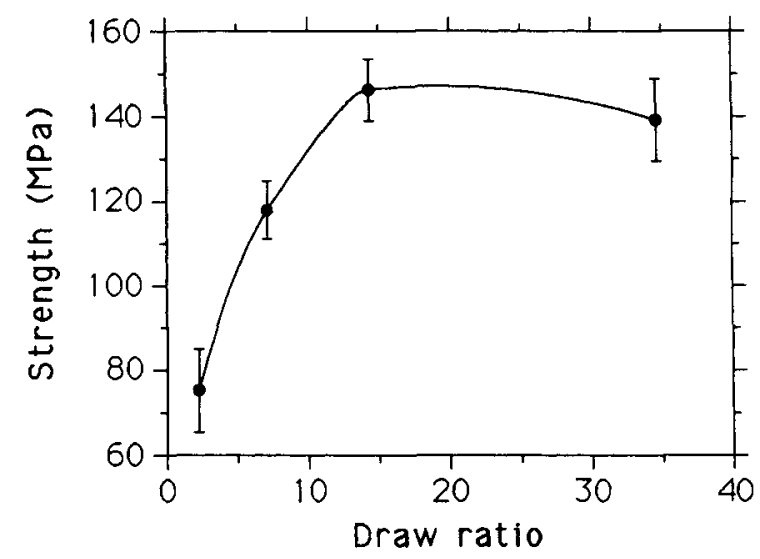

Fig. 15. Variation of tensile strength with draw ratio for Lexan 151 / Vectra B950 (30 wt\% LCP) composite strands.

ing draw ratio up to -15 . The highest value of tensile strength is $146 \mathrm{MPa}$. Again this increment results mainly form LCP molecular orientation. The tensile strength of the strand with a draw ratio of 35 falls slightly. This decrement is due largely to a variance in the gripping method when the samples were tested. Because of the small diameter of the samples with higher draw ratios, it was very difficult to determine the elongation for these samples with an extensome- 
ter. Therefore, strain rates were measured by the position and speed of the crosshead. In addition, sandpaper was used to hold the sample instead of rubber pads to avoid the extra elongation resulting from rubber deformation. This change in gripping method produced high stress concentrations at both grips. Consequently a drop in tensile strength ensued.

All samples reported herein broke in a brittle mode despite the high ductility of the PC matrix. No necking was detected, and the elongation to break was less that $2 \%$. LCP fibril formation and the interface formed therefrom are probably responsible for this embrittlement effect.

To summarize, in-situ composite strands of PC/V30 have been fabricated with elastic modulus as high as $19 \mathrm{GPa}$ and tensile strength $146 \mathrm{MPa}$, with draw ratios below 40. Compared with the thermoplastic matrix, the improvements in elastic modulus and tensile strength are 8.3 times (19 GPa vs. $2.3 \mathrm{GPa}$ ) and 2.4 times (146 $\mathrm{MPa}$ vs. $60 \mathrm{MPa}$ ), respectively, with the addition of only $30 \mathrm{wt} \%$ LCP. These improvements are attributed to microfibril formation of the LCP phase, and more importantly to LCP molecular orientation. Based on these understandings, a composite model has been proposed to account for the change of elastic modulus of the reinforcing LCP phase with draw ratio in in-situ composite strands. The LCP phase is envisaged as a composite of perfectly oriented chains and randomly oriented chains. Equations for longitudinal and transverse elastic moduli of in-situ composite strands are then developed based on the well-known Halpin-Tsai equation and the composite model of the reinforcing LCP phase. Theoretical predictions of longitudinal elastic moduli agree fairly well with experimental results for the polycarbonate/Vectra composites. Details of the model will be presented in a future paper (51).

\section{CONCLUSIONS}

A composite material with well-defined microfibrils of Vectra B950 LCP in Lexan 151 bisphenol-A PC matrix has been successfully fabricated by extrusion followed by a drawing process with a co-rotating twin-screw extruder. The resulting in-situ composites have elastic modulus as high as $19 \mathrm{GPa}$ and tensile strength $146 \mathrm{MPa}$ with draw ratios below 40 and LCP concentration of $30 \mathrm{wt} \%$. Compared with the thermoplastic matrix, the elastic modulus and tensile strength of the in-situ composite have increased by 7.3 times and 1.4 times, respectively, with the addition of only $30 \mathrm{wt} \% \mathrm{LCP}$.

Drawing has a paramount effect on the structure and hence the properties of the composite strands. Drawing not only is a necessary procedure for microfibril formation, but also promotes LCP crystallization and molecular orientation in the composites. As a result, mechanical properties are significantly improved by drawing. It has been found that both the crystallite size and the heat of fusion of LCP increase constantly with draw ratio. However, the crystal- nematic transition temperature of LCP and glass transition temperature of the PC matrix are virtually unaffected by drawing. These results are ascribed to the (semi)rigid rod nature of the LCP and the persistence of an ordered structure in the LCP melt. Mechanical properties (modulus and tensile strength) rise with draw ratio for the composite fibers with 30 wt\% LCP content. This improvement is attributed to fibrillation of the LCP phase in the blend and the increasing orientation of the LCP chains along the fiber axis during drawing.

\section{ACKNOWLEDGMENTS}

The authors would like to thank Dr. Hristo Hristov for his valuable assistance in orientation parameter measurements.

\section{REFERENCES}

1. G. Kiss, Polym. Eng. Sci., 27, 410 (1987).

2. B. Bassett and A. F. Yee, Polym. Compos., 11, 10 (1990).

3. D. G. Baird and R. Ramanathan, in Contemporary Topics in Polymer Science, Vol. 6, p. 73, Multiphase Macromolecular Systems, B. M. Culbertson, ed., Plenum, New York (1988).

4. R. A. Weiss, W. Huh, and L. Nicolais, in High Modulus Polymers, p. 145, A. Z. Zachariades and R. S. Porter, eds., Marcel Dekker, New York (1988).

5. D. Dutta, F. Fruitwala, A. Kohli, and R. A. Weiss, Polym. Eng. Sci., 30, 1005 (1990).

6. W. Brostow, Polymer, 31, 979 (1990).

7. G. Crevecoeur and G. Groeninckx, Bull. Soc. Chim. Belg., 99 (11-12), 1031 (1990).

8. R. A. Weiss and C. K. Ober, Liquid Crystalline Polymers, ACS Symp. Series 435 (1990).

9. V. G. Kulichikhin and N. A. Plate, Polym. Sci., U.S.S.R., 33, 1 (1991).

10. A. Siegmann, A. Dagan, and S. Kenig, Polymer, 26. 1325 (1985).

11. P. J. Flory, Macromolecules, 11, 1138 (1978).

12. J. C. Halpin and S. W. Tsai. Effect of Environmental Factors on Composite Materials, AFML-TR 67-423 (June 1969).

13. G. I. Taylor, Proc. R. Soc. London, Ser-A, 146, 501 (1934).

14. H. B. Chin and C. D. Han, J. Rheol, 23, 557 (1979).

15. M. Tsebrenko, N. Rezanera, and G. Vinnogradov, Polym. Eng. Sci., 20, 1023 (1980).

16. J. X. Li, M. S. Silverstein, A. Hiltner, and E. Baer, J. Appl Polym. Sci., 44, 1531 (1992).

17. K. G. Blizard and D. G. Baird, Polym. Eng. Sci., 27, 653 (1987).

18. A. M. Sukhadia, D. Done, and D. G. Baird, SPE ANTEC Tech. Papers, 35, 1847 (1989).

19. R. J. Amundsen, Master's thesis, The University of Michigan, Ann Arbor, Mich. (1990).

20. A. Kohli, N. Chung, and R. A. Weiss, Polym. Eng. Sci., 29, 573 (1989).

21. A. I. Isayev and M. J. Modic, Polym. Compos., 8, 158 (1987).

22. D. Beery, S. Kenig, and A. Siegmann, Polym. Eng. Sci., 31, 451 (1991).

23. D. Acierno, E. Amendola, C. Carfagna, L. Nicolais, and M. R. Nobile, Molec. Cryst., Liq, Cryst., 153, 553 (1987).

24. K. G. Blizard, C. Federici, O. Federico, and L. L. Chapoy, Polym. Eng. Sci., 30, 1442 (1990).

25. T. Katoh and N. R. Schott, SPE ANTEC Tech. Papers, 36, 1861 (1990).

26. S. Kenig, Polym Adv. Technol 2, 201 (1992).

27. H. J. O'Donell, H. H. Chen, A. A. Handlos, A. Datta, and D. G. Baird, ACS Polymer Preprints, 33 (1), 376 (1992). 
28. L. M. Mallagh, E. R. Rouyer, I. S. Miles, and W. A. MacDonald, Third European Symposium on Polymer Blends, D14, Cambridge, England (July 1990).

29. T. Sun, D. G. Baird, H. H. Huang, D. S. Done, and G. L. Wilkes, J. Compos. Mater., 25, 789 (1991).

30. Hoechst-Celanese Co., Vectra B950 Safety Data Sheet (1991).

31. G. Crevecoeur and G. Groeninckx, Polym. Eng. Sci., 30, 532 (1990).

32. F. P. La Mantia and A. Valenza, Makromol Chem., Macromol. Symp., 38, 183 (1990).

33. C. D. Han, Multiphase Flow in Polymer Processing, Academic Press, New York (1981).

34. Q. Lin, J. Jho, and A. F. Yee, to be published.

35. R. L. Miller, Flow-Induced Crystallization in Polymer Systems, Gordon and Breach Science Publishers, New York (1979).

36. G. Eder, H. Janeschitz-Kriegl, and S. Liedauer, Prog. Polym. Sci., 15, 629 (1990).

37. J. Blackwell and A. Biswas, Developments in Oriented Polymers, Vol. 2, p. 153, I. M. Ward, Elsevier Applied Science, London (1987).

38. H. Tadokoro. Structure of Crystalline Polymers, John Wiley \& Sons, New York (1979).
39. A. Guinier, X-Ray Diffraction in Crystals, Imperfect Crystals, and Amorphous Bodies, W. H. Freeman and Co., San Francisco (1963).

40. A. Peterlin, Crystallization Phenomena, in ref. 33, p. 1.

41. J. Brandrup and E. H. Immergut, Polymer Handbook, 2nd ed., John Wiley \& Sons, New York (1975).

42. T. S. Chung, J. Polym. Sci., Polym Phys. Ed., 26, 1549 (1988).

43. S. Kenig, Polym. Eng. Sci., 27, 877 (1987); 29, 1136 (1989).

44. I. M. Ward, Adv. Polym. Sci., 66, 81 (1985).

45. L. E. Alexander, X-Ray Diffraction Methods in Polymer Science, John Wiley and Sons, New York (1969).

46. S. H. Jung and S. C. Kim. Polym. J. 20, 73 (1988).

47. T. S. Chung, Plast. Eng., October 1987, p. 39.

48. A. Ajji, J. Brisson, and Y. Qu, J. Polym Sci., Polym. Phys. Ed., 30, 505 (1992).

49. Ginghuang Lin and A. F. Yee, Am. Chem. Soc., Div. Polym. Chem. Polym. Prepr., 33 (1), 298 (1992).

50. C. Carfagna, E. Amendola, L. Nicolais, D. Acierno, $O$. Francescangeli, B. Yang, and F. Rustichelli, J. Appl. Polym Sci., 43, 839 (1991).

51. Qinghuang Lin and A. F. Yee, to be published. 